\title{
Evidence for radiological and histopathological prognostic importance of detecting extramural venous invasion in rectal cancer: recommendations for radiology and histopathology reporting
}

\section{Introduction}

Extramural venous invasion (EMVI) is widely regarded as an adverse prognostic feature in rectal cancer. Whilst the effect of venous invasion has been studied for more than seven decades, Talbot's study from St Mark's Hospital in 1980 was the first to specify the importance of extramural $v s$ intramural venous invasion and show that it was only invasion of tumour into the extramural veins that had a prognostic effect [1]. However, the current literature is somewhat confusing, which is most probably due to heterogeneity of definitions. We discuss some of the available evidence and offer guidance on the optimal criteria for both histopathological and radiological detection of EMVI within the context of modern management of rectal cancer.

\section{Prevalence}

The true prevalence of histopathological EMVI in rectal cancer is difficult to know and hinges on the rigour of macroscopic and microscopic pathological evaluation, and whether ancillary techniques have been used. Reported detection rates have been inconsistent and the incidence ranges from 9 to $61 \%[1-12]$. Some series have shown selection bias towards advanced cases which may affect the true prevalence and result in a high EMVI detection rate [13], whilst others do not differentiate between rectal and colonic tumours. A more recent challenge has been that the increasing use of preoperative radiotherapy has led to an inherent false-negative rate, resulting in under-reporting of venous invasion due to obliteration of normal venous architecture when involved veins are destroyed beyond recognition [14].

\section{Histopathology-based detection}

\section{Optimal macroscopic examination}

Rectal cancer resection specimens must be appropriately opened, pinned out and fixed in formalin solution for at least $48 \mathrm{~h}$ before dissection. Once adequately fixed, the specimen should be cross-sectionally sliced trans- versely at a thickness of 3-4 $\mathrm{mm}$; these thin slices allow identification of the depth of tumour invasion and status of the circumferential resection margin. In addition, serpiginous outgrowths of tumour extending beyond and at right angles to the muscularis propria may be visible and probably represent a focus of EMVI (Fig. 1). Areas for microscopic examination are then selected with at least four sections of tumour taken targeting areas of greatest interest. Evidence suggests that sections taken perpendicular to the tumour at the area of maximal invasion are most likely to identify EMVI [15]. As many rectal tumours will have undergone neoadjuvant radiotherapy, it may be difficult to identify areas of residual tumour; in this instance a greater number of blocks may be required concentrating on areas of fibrosis in the region of the irradiated tumour.

\section{Optimal microscopic examination}

The microscopic identification of EMVI relies upon the identification of tumour cells within an endothelial or smooth muscle-lined space containing red blood cells outside the muscularis propria of the bowel wall as defined in the UK Royal College of Pathologists (RCPath) guidelines for reporting colorectal cancer

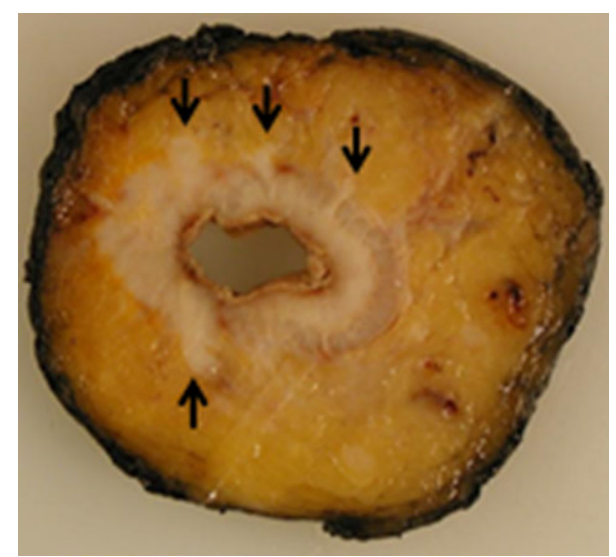

Figure I Gross pathology specimen showing tumour with EMVI. Intravascular tumour deposits can be seen as serpigenous extensions of tumour projecting from and at right angles to the muscularis propria (see arrows). 


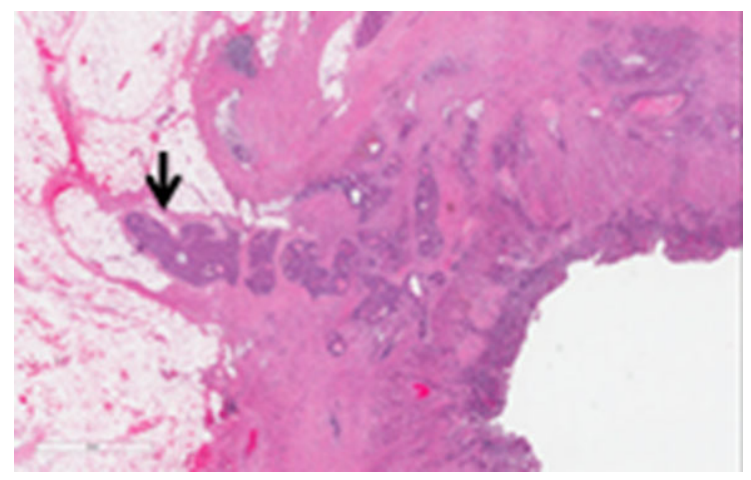

Figure 2 Micrograph showing venous invasion as a linear extension of tumour projecting beyond the muscularis propria (see arrow). The vessel wall is largely destroyed by tumour and an appreciation of the characteristic architecture is required to accurately recognize this phenomenon.

[16]. Due to the difficulties associated with tissue sectioning and architectural distortion by tumour or fibrosis, vascular spaces may become difficult to identify on routine haematoxylin and eosin (H\&E) staining (Fig. 2). As a result the rate of EMVI with H\&E staining has been documented to be as low as 8\% [17]; however, the RCPath guidelines suggest that EMVI should be detected in at least $25 \%$ of cases of colorectal cancer. There is no guidance as to the expected rate in rectal cancers alone or those treated with neoadjuvant radiotherapy; however, $25 \%$ may still be an underestimate for the true prevalence of EMVI using an optimal sampling technique. There is poor agreement, even between specialist gastrointestinal histopathologists, when identifying vascular invasion using $H \& E$ [18]. In situations where uncertainty or disagreement exists as to the nature of subserosal tumour deposits, the RCPath guidelines recommend that deeper sections through the tissue block should be performed to attempt to clarify the nature of a lesion; alternatively special histochemical and immunohistochemical stains can be invaluable tools.

\section{Special stains: histochemical staining}

The elastic lamina within medium-to-large vessels provides a landmark, which can be highlighted by special stains such as orcien and elastin Van Gieson (EVG); the application of these stains has been shown to increase the detection of EMVI from $19.6 \%$ to $58 \%$ [19]. Staining for the elastic lamina also improves agreement between pathologists from poor to fair [18]. The only significant drawback of these methods is that in selected cases interpretation may be hampered by background staining although this is rare.
Figure 3 Extramural venous invasion demonstrated on H\&E staining (a) and confirmed using elastin Van Gieson (b), and antibodies against smooth muscle actin (c), CD34 (d) and CD31 (e). The position of the tumour within the vessel is highlighted by an arrow.
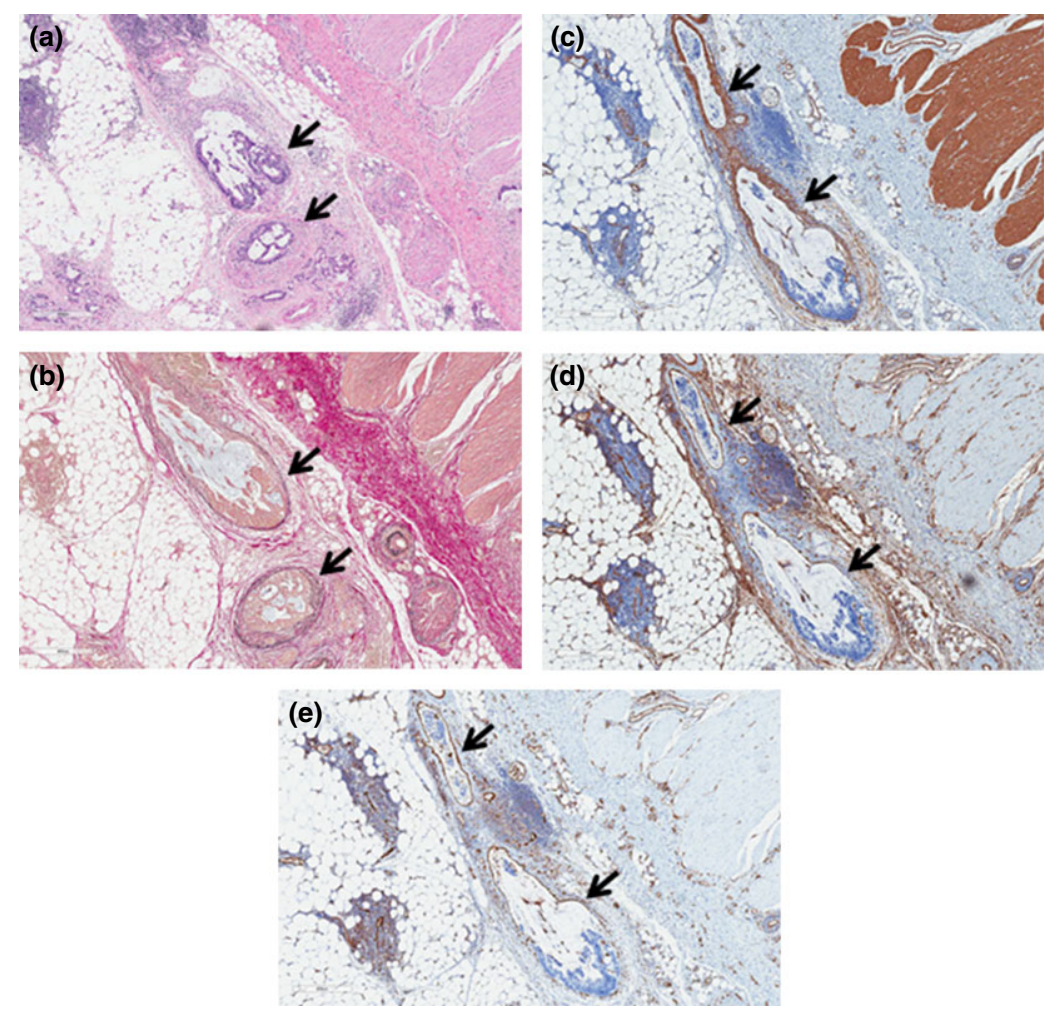


\section{Special stains: immunohistochemistry}

Immunohistochemical stains using antibodies directed towards endothelial (CD31 and CD34) or smooth muscle (smooth muscle actin) antigens within vessel walls also increase the sensitivity of histopathologydetected EMVI. The potential advantage of these methods is an increased specificity over histochemical staining, although the endothelium may become lost or obscured when a tumour embolus occupies a vessel.

Kingston et al. examined the rate of detection when using H\&E, EVG and immunohistochemistry for CD31 and CD34 in 50 cases of colorectal cancer in which vascular invasion had previously not been identified; with the use of special stains they identified vascular invasion in $48 \%$ of cases [20]. Although this study did not differentiate between intramural and extramural vascular invasion, it showed that EVG was the most sensitive method; however, in a minority of cases immu- nohistochemical staining was still required. An example of these histochemical and immunohistochemical stains in practice is shown in Fig. 3a-e.

\section{MRI-based detection}

\section{Optimal technique}

High-resolution MRI is accurate in detecting EMVI (mrEMVI) both before and after preoperative therapy. Accurate detection is underpinned by strict adherence to technique and image acquisition as well as a threedimensional understanding of the vascular anatomy surrounding the rectum. Failure of the latter can confuse venous invasion with nodal disease. Most scanners will provide adequate $3 \mathrm{~mm}$ slices; however, optimal technique involves ensuring appropriate an field of view $(\mathrm{FOV})(16 \times 16 \mathrm{~cm})$ to obtain high-resolution images. Incorrect FOV also results in loss of voxel size and ultimately resolution. Furthermore, the field alignment
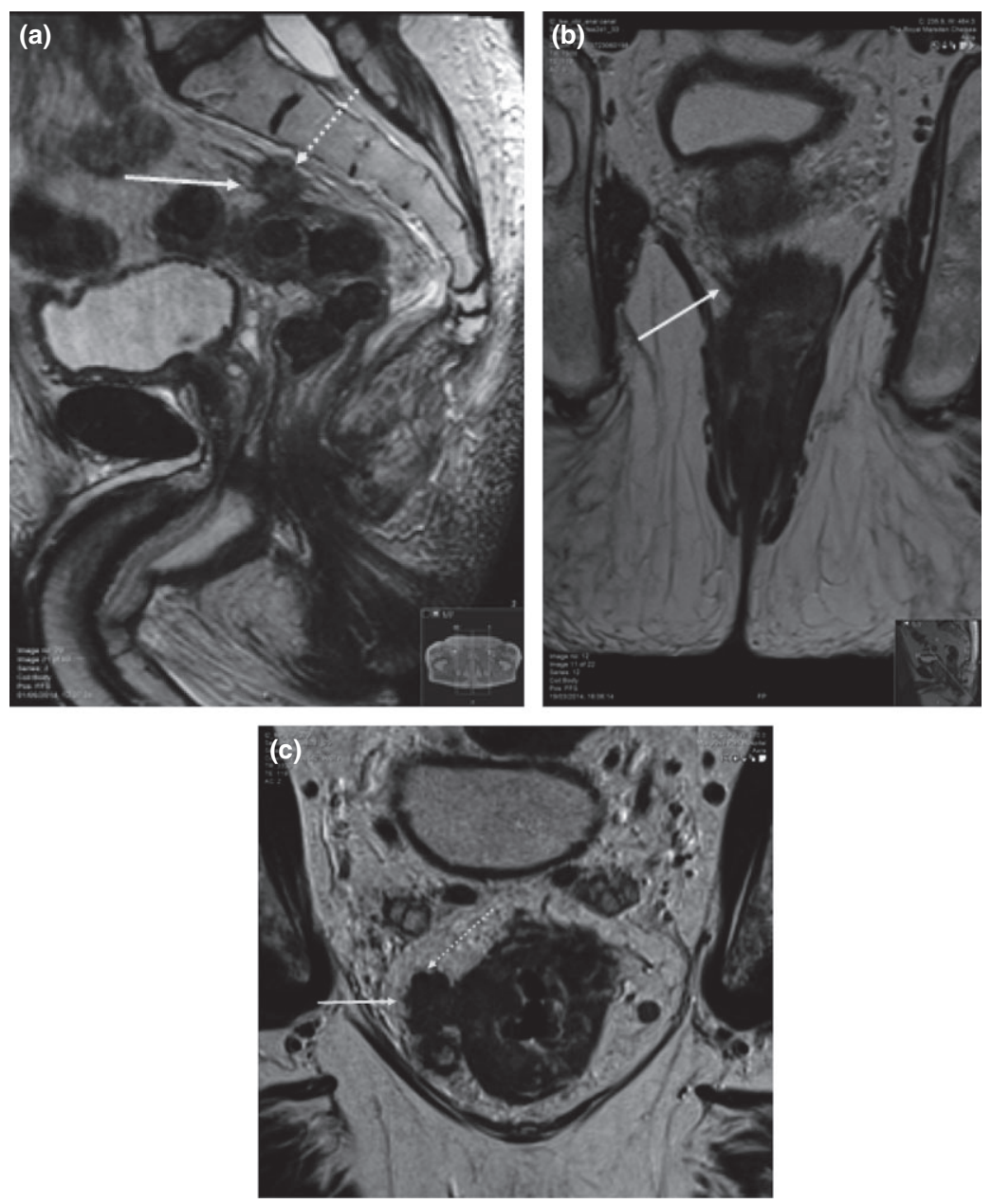

Figure 4 (a) MRI showing tumour invasion into the superior rectal vein. Dashed arrow indicates tumour extension. Solid arrow shows signal void in vessel which is surrounded by tumour. (b) MRI showing tumour extending into the middle rectal vein. The dashed arrow shows signal void (black) shown in the middle rectal vein; the solid arrow shows tumour extension beyond the muscularis (c) MRI showing tumour in the inferior rectal vein. There is signal void which could be followed on sequential images confirming this was the inferior rectal vein. 
must be perpendicular to the long axis of the rectum or vessels can be missed.

\section{Characteristic radiological features of EMVI}

Veins around the rectum are recognized on T2weighted images as serpiginous or tortuous linear structures which appear black due to the signal void of blood. The larger 'named' vessels such as the superior and middle rectal veins appear with anatomical consistency, which helps in confident identification. Detection of mrEMVI relies on the observation of intermediate tumour signal disrupting the normal configuration of the vessel resulting in irregular expansion of the vein, loss of the signal void and intermediate signal intensity which may or may not be contiguous with the main tumour in the rectal wall [21]. Smaller venules can be seen perforating the normal outer rectal wall and produce a low to intermediate signal intensity in tubular structures on T2-weighted images - these are normal vessels and should not be confused with mrEMVI [22]. Venous invasion into these smaller venules can be recognized by their expansion and irregularity adjacent to the tumour due to contiguous tumour extension (Fig. $4 \mathrm{a}-\mathrm{c})$.

\section{Early studies showing correlation with histopathology}

The first study to demonstrate the accuracy of MRI in identifying the EMVI as a prognostic feature of rectal cancer involved 98 patients with biopsy-proven rectal cancer [23]. The prognostic significance of mrEMVI has further been documented in a study by Smith et al. [24]. They offered a scoring system based on previous experience which identified particular morphological and signal characteristics. Recurrence-free survival at 3 years was compared between MRI and histopathology-detected EMVI and was reported as $35 \%$ and $34 \%$, respectively. Recurrence-free survival when EMVI was not present was $73.8 \%$ and $74.1 \%$ respectively. A further study using similar grading criteria reported mrEMVI in $42 \%$ of patients undergoing primary surgery for rectal cancer. An mrEMVI score of 3-4 had 100\% sensitivity and $89 \%$ specificity in identifying EMVI involving veins greater than $3 \mathrm{~mm}$ in diameter [25].

\section{The potential of mrEMVI as an imaging biomarker}

The prognostic effect of traditional adverse features has not been prospectively validated in patients who have undergone preoperative therapy. Perhaps those features which we previously understood as having a significant bearing on survival outcomes may not be as important following chemoradiotherapy (CRT) providing optimal surgery has taken place - such as nodal disease [26].

MRI is accurate in identifying EMVI following chemoradiation providing the same diagnostic standard is used $[27,28]$. Indeed, a comparison between the outcomes of patients with mrEMVI and pathologydetected EMVI showed that, not only is it associated with poor disease-free survival when either technique is used, but MRI identified more cases of EMVI than pathology following CRT [29]. Further, a recent study involving Stage II and Stage III patients who had undergone preoperative long-course CRT showed that evidence of EMVI led to significantly worse disease recurrence and in fact Stage II tumours with EMVI had similar outcomes to Stage III tumours [30]. mrEMVI has also been shown to be a potential imaging biomarker following neoadjuvant CRT. EMVI can be graded following CRT using a regression grading score based on the degree of radiological evidence of fibrosis shown and this can be linked to survival outcomes. Patients who demonstrated fibrosis of more than $50 \%$ in EMVI had a 3 -year disease-free survival of $87.9 \%$. Where there was less than $50 \%$ fibrosis disease-free survival was $45.8 \%$. Fibrosis of less than $50 \%$ had a hazard ratio of 5.75 for disease recurrence [31].

\section{Conclusion}

There is now substantial evidence suggesting that EMVI is an adverse prognostic feature in rectal cancer, including in cases treated with neoadjuvant therapy. Whilst special stains undoubtedly increase the reporting rates, $H \& E$ staining unfortunately remains the gold standard for most institutions. The use of special stains will improve detection rates particularly following CRT and must surely now be considered as routine in addition to meticulous technique.

Furthermore, MRI can identify EMVI at the same rate as histopathology and predicts the same outcome. Using the radiological criteria described above will allow for more consistent reporting of EMVI in the preoperative setting. Multidisciplinary teams are urged to regularly audit the reporting rates of EMVI using both MRI and histopathology to ensure good concordance.

\section{Acknowledgement}

NIHR BRC Programme Grant.

\section{Conflicts of interests}

None. 


\section{Author contributions}

All authors contributed equally to analysis and writing of the paper. Conception was between Professor Brown and $\mathrm{Mr}$ Chand. Image collection was equally done by all authors.

\section{Chand*, T. Palmer†, L. Blomqvistł, I. Nagtegaal§̧, N. West $\llbracket$ and G. Brown**}

*Royal Marsden Hospital, Downs Road, Sutton, SM2 5PT, UK; †Imperial College London, Exhibition Road, London, UK; †Karolinska University Hospital, Karolinska, Sweden; §Radboud University Medical Centre, Nijmegen, The Netherlands; q Leeds University Hospital, Leeds, UK and ***Department of Radiology, Royal Marsden Hospital, Sutton, UK E-mail: Manish.chand@rmh.nhs.uk

\section{References}

I Talbot IC, Ritchie S, Leighton MH, Hughes AO, Bussey $\mathrm{HJ}$, Morson BC. The clinical significance of invasion of veins by rectal cancer. Br J Surg 1980; 67: 439-42.

2 Brown CF. Visceral metastasis from rectal carcinoma. Surg Gynecol Obstet 1938; 66: 611-21.

3 Dukes CE, Bussey HJ. The spread of rectal cancer and its effect on prognosis. Br J Cancer 1958; 12: 309-20.

4 Seefeld PH, Bargen JA. The spread of carcinoma of the rectum: invasion of lymphatics, veins and nerves. Ann Surg 1943; 118: 76-90.

5 Madison MS, Dockerty MB, Waugh JM. Venous invasion in carcinoma of the rectum as evidenced by venous radiography. Surg Gynecol Obstet 1954; 92: 170-8.

6 Khankhanian N, Mavligit GM, Russell WO, Schimek M. Prognostic significance of vascular invasion in colorectal cancer of Dukes' B class. Cancer 1977; 39: 1195-200.

7 Heald RJ, Ryall RD. Recurrence and survival after total mesorectal excision for rectal cancer. Lancet 1986; 1: 1479-82.

8 Rich T, Gunderson LL, Lew R, Galdibini JJ, Cohen AM, Donaldson G. Patterns of recurrence of rectal cancer after potentially curative surgery. Cancer 1983; 52: $1317-29$.

9 Jass JR, Atkin WS, Cuzick J et al. The grading of rectal cancer: historical perspectives and a multivariate analysis of 447 cases. Histopathology 1986; 10: 437-59.

10 Sasaki O, Atkin WS, Jass JR. Mucinous carcinoma of the rectum. Histopathology 1987; 11: 259-72.

11 Minsky BD, Mies C, Recht A, Rich TA, Chaffey JT. Resectable adenocarcinoma of the rectosigmoid and rectum. II. The influence of blood vessel invasion. Cancer 1988; 61: 1417-24.

12 Dukes CE, Bussey HJ. Venous spread in rectal cancer. Proc $R$ Soc Med 1941; 34: 571-3.

13 Vass DG, Ainsworth R, Anderson JH, Murray D, Foulis $\mathrm{AK}$. The value of an elastic tissue stain in detecting venous invasion in colorectal cancer. J Clin Pathol 2004; 57: 769 72 .
14 Sternberg A, Amar M, Alfici R, Groisman G. Conclusions from a study of venous invasion in stage IV colorectal adenocarcinoma. J Clin Pathol 2002; 55: 17-21.

15 Sternberg A, Mizrahi A, Amar M, Groisman G. Detection of venous invasion in surgical specimens of colorectal carcinoma: the efficacy of various types of tissue blocks. J Clin Pathol 2006; 59: 207-10.

16 Williams GT, Quirke P, Shepherd NA. Dataset for Colorectal Cancer, 2nd edn, 2007. Available from http:// www.rcpath.org/resources/pdf/G049-ColorectalDatasetSep07.pdf. (accessed August 2014)

17 West NP, Hohenberger W, Weber K, Perrakis A, Finan PJ, Quirke P. Complete mesocolic excision with central vascular ligation produces an oncologically superior specimen compared with standard surgery for carcinoma of the colon. J Clin Oncol 2010; 28: 272-8.

18 Kirsch R, Messenger DE, Riddell RH et al. Venous invasion in colorectal cancer: impact of an elastin stain on detection and interobserver agreement among gastrointestinal and nongastrointestinal pathologists. Am J Surg Pathol 2013; 37: 200-10.

19 Roxburgh CS, McMillan DC, Anderson JH, McKee RF, Horgan PG, Foulis AK. Elastica staining for venous invasion results in superior prediction of cancer-specific survival in colorectal cancer. Ann Surg 2010; 252: 989-97.

20 Kingston EF, Goulding H, Bateman AC. Vascular invasion is underrecognized in colorectal cancer using conventional hematoxylin and eosin staining. Dis Colon Rectum 2007; 50: $1867-72$.

21 Smith NJ, Shihab O, Arnaout A, Swift RI, Brown G. MRI for detection of extramural vascular invasion in rectal cancer. AJR Am J Roentgenol 2008; 191: 1517-22.

22 Brown G, Kirkham A, Williams GT et al. High-resolution MRI of the anatomy important in total mesorectal excision of the rectum. AJR Am J Roentgenol 2004; 182: $431-9$.

23 Brown G, Radcliffe AG, Newcombe RG, Dallimore NS, Bourne MW, Williams GT. Preoperative assessment of prognostic factors in rectal cancer using high-resolution magnetic resonance imaging. Br J Surg 2003; 90: 355-64.

24 Smith NJ, Barbachano Y, Norman AR, Swift RI, Abulafi AM, Brown G. Prognostic significance of magnetic resonance imaging-detected extramural vascular invasion in rectal cancer. Br J Surg 2008; 95: 229-36.

25 Koh DM, Smith NJ, Swift RI, Brown G. The relationship between MR demonstration of extramural venous invasion and nodal disease in rectal cancer. Clin Med Oncol 2008; 2: 267-73.

26 Chand M, Heald RJ, Brown G. The importance of not overstaging mesorectal lymph nodes seen on MRI. Colorectal Dis 2013; 15: 1201-4.

27 Patel UB, Brown G, Rutten H et al. Comparison of magnetic resonance imaging and histopathological response to chemoradiotherapy in locally advanced rectal cancer. Ann Surg Oncol 2012; 19: 2842-52.

28 Yu SK, Tait D, Chau I, Brown G. MRI predictive factors for tumor response in rectal cancer following neoadjuvant 
chemoradiation therapy - implications for induction chemotherapy? Int J Radiat Oncol Biol Phys 2013; 87: 505-11.

29 Chand M, Evans J, Swift RI et al. The prognostic significance of postchemoradiotherapy high-resolution MRI and histopathology detected extramural venous invasion in rectal cancer. Ann Surg 2015; 261: 473-9.

30 Chand M, Bhangu A, Wotherspoon A et al. EMVI-positive stage II rectal cancer has similar clinical outcomes as stage
III disease following pre-operative chemoradiotherapy. Ann Oncol 2014; 25: 858-63.

31 Chand M, Swift RI, Tekkis PP, Chau I, Brown G. Extramural venous invasion is a potential imaging predictive biomarker of neoadjuvant treatment in rectal cancer. $\mathrm{Br} J$ Cancer 2014; 110: 19-25. 